\title{
The impact of Endocuff-assisted colonoscopy on adenoma detection in an organized screening program
}

\section{다 (우우}

\author{
Authors \\ Claudia Roldo ${ }^{3}$, Fabio Soppelsa ${ }^{4}$, Bastianello Germanà' \\ Institutions \\ 1 Gastroenterology Unit, San Martino Hospital, ULSS 1, \\ Belluno, Italy \\ 2 Gastroenterology Unit, Nuovo Regina Margherita \\ Hospital, Rome Italy \\ 3 Pathology Unit, San Martino Hospital, ULSS 1, Belluno \\ Italy \\ 4 Public Health Service, San Martino Hospital, ULSS 1, \\ Belluno, Italy
}

Lucas G. Cavallaro' ${ }^{1}$, Cesare Hassan², Pierenrico Lecis ${ }^{1}$, Ermenegildo Galliani ${ }^{1}$, Elisabetta Dal Pont ${ }^{1}$, Paolo luzzolino ${ }^{3}$,

submitted 16.10 .2017

accepted after revision 2.2.2018

\author{
Bibliography \\ DOI https://doi.org/10.1055/a-0578-8515 | \\ Endoscopy International Open 2018; 06: E437-E442 \\ (c) Georg Thieme Verlag KG Stuttgart · New York \\ ISSN 2364-3722
}

Corresponding author

Lucas G. Cavallaro, Gastroenterology Unit, San Martino Hospital ULSS 1, Viale Europa 22, 32100 Belluno, Italy Fax: +390437516580

cavallarolucas@libero.it

\section{ABSTRACT}

Background and study aims Colorectal cancer (CRC) screening with biennial fecal occult blood test has been shown to reduce CRC mortality. For the effectiveness of the CRC screening program is crucial that a high-quality colonoscopy with a high adenoma detection rate (ADR) be performed. To improve ADR, various endoscopic devices have been developed. Endocuff, an endoscopic cap with finger-like projections, has been shown to improve ADR. The aim of this study was to compare in an organized CRC screening program ADR, advanced adenoma detection rate (AADR) and mean number of adenomas per patient (MAP) using standard colonoscopy (SC) and Endocuff-assisted colonoscopy (EAC).

Patients and methods We compared performance of SC (in 2014) and EAC (in 2015) in consecutive participants in an organized CRC screening program.

Results SC and EAC were performed in 546 (284 males) and 519 (293 males) subjects, respectively (mean age 60 years). Cecal intubation rate was $97.4 \%$ for SC and $97.1 \%$ for EAC and not significantly different $(P=0.7)$. ADR was $47 \%$ for SC and $52 \%$ for EAC, $P=0.1$. MAP in SC and EAC were 0.87 (range: $0-7$ ) and 1.11 (range: $0-13$ ) respectively, $P=0.02$. AADR rate was $25 \%$ and $23 \%$ for SC and EAC, respectively, $P=0.5$.

Conclusion Endocuff-assisted colonoscopy does not improve the number of patients with at least one adenoma but it may increase the number of detected adenomas per procedure.

\section{Introduction}

Colorectal cancer (CRC) is the second most common cause of death among cancers [1]. CRC screening with biennial fecal occult blood test has been shown to reduce CRC mortality [2-4]. Over the last 15 years, national screening programs have been initiated in many European countries, including Italy [5]. In the CRC screening setting, colonoscopy, which is performed when fecal test results positive, is an extension of the program [6]. For the effectiveness of the CRC screening program it is crucial that a high-quality colonoscopy with a high adenoma detection rate (ADR) be performed [7]. A high ADR is, in fact, the major predictor of lower CRC risk and cancer-related mortality [8].

To optimize the proportion of the observed mucosal surface and, consequently the ADR, several devices and technologies have been introduced [9].

The Endocuff (Arc Medical Design Ltd, Leeds, England) is a device composed of a soft, cylindrical, polymer with flexible projections arranged circumferentially. It is mounted onto the distal tip of the colonoscope without impairing both the view and function of the instrument. During the withdrawal phase the hinged projections extend radially, flattening the colonic 
mucosal folds and, potentially, improving mucosal visualization [10].

In two prospective randomized multicenter trials, Biecker et al. and Floer et al. in a mixed population (colonoscopies performed for screening, surveillance and symptomatic subjects) reported increased detection of both polyps and adenomas when Endocuff was used. In particular, they observed smaller and more sessile detected polyps by means of the device without differences in terms of cecal intubation rate, procedural time or significant adverse events (AEs) $[11,12]$. Conversely, Van Doorn et al., in a multicenter randomized controlled trial, did not observe any difference in ADR between Endocuff-assisted colonoscopy (EAC) and standard colonoscopy (SC) but reported a higher number of diminutive/small adenomas when Endocuff was used [13]. In accordance, recently, a randomized controlled trial in a CRC screening program, based on fecal occult blood test, demonstrated no difference in terms of detection rate between the two techniques [14]. Otherwise, other two studies, performed outside the CRC screening setting, showed a significantly higher adenoma detection rate for EAC than for SC $[15,16]$.

The aim of this study was to exam, in a retrospective cohort, the effect of use of Endocuff on adenoma detection in a FITbased population program. In particular, all CRC screening colonoscopies in 2015 were carried out with Endocuff and their outcomes were compared with those for the year 2014 during which Endocuff was not used.

\section{Patients and methods}

\section{Patients}

Since 2005 our Local Health Unit (LHU-1) located in the Veneto Region (North-eastern of Italy) has been offering asymptomatic residents aged 50 to 69 CRC screening based on biennial fecal immunochemical test (FIT) (1st level) and, if FIT-positive, a colonoscopy (2nd level). This program is a part of the Veneto Region population-based CRC screening programs [5]. LHU-1 population globally consists of 124,710 inhabitants. In 2014, the target population for CRC screening (aged 50 to 69 years) was 36,023 inhabitants. The rate of extension of invitations (invited persons/target population) for FIT was $90 \%$ with a $60.2 \%$ of adherence (adherent persons/invited persons) and $4 \%$ FITpositives. The subjects were consecutively enrolled by the LHU-1 Department of Health during 2014 and 2015. They were offered colonoscopy: (a) if they were both asymptomatic and FIT-positive; (b) as surveillance of adenomas previously removed during a colonoscopy in the CRC screening program; or (c) to complete a colonoscopy (in previously FIT-positives patients) due to previous poor preparation or to reach the cecum in a previous uncompleted colonoscopy. If the subjects were offered more than one colonoscopy during the studied period, only the first one was included in this study.

\section{Screening colonoscopy}

All colonoscopies in the LHU-1 CRC screening program were performed in our unit. A colonoscopy journey per week was devoted to the CRC screening and 14 scheduled screening exams were carried out during this session. Olympus Colonoscopes (CF-H180AI and CF-165I instruments, Olympus Medical Systems, Tokyo, Japan) were used. Carbon dioxide pumps were used for insufflation. Exams were performed under moderate sedation (meperidine and midazolam intravenously) or without. The exception was colonoscopy with deep anaesthesia, which was done with an anesthesiologist administering propofol. Low-volume polyethylene glycol (PEG) bowel preparation (Moviprep, Norgine, Amsterdam, Netherlands) in a split-dose regimen was recommended with the second half dose taken 6 hours before the procedure as well as a diet without high-fiber foods 3 days before. Intestinal preparation per patients was scored according to the Boston Bowel Preparation Score (BBPS). In the study, SC and EAC were performed by the same team of endoscopists (LGC, ED, EG, BG, PL) who performed at least 1000 colonoscopies. Withdrawal time was recorded by a member of the research staff using a stopwatch. It was measured in all completed diagnostic colonoscopies with good bowel preparation [7].

\section{Endocuff-assisted colonoscopy}

For all screening colonoscopies performed from 1 January to 31 December 2015, the first version of Endocuff with one proximal and one distal row of finger-like projections was used. Use of Endocuff was explained to the endoscopists with an information leaflet and/or with the assistance of an explanatory video. The device was placed snugly around the colonoscope tip by the endoscopy nurse before insertion and held on by friction. The investigations reported in the manuscript were performed with written informed consent before participation and followed all the guidelines for experimental investigation with human subjects according to the Helsinki declaration. The study was approved by the CRC screening board of the LHU-1.

\section{Histopathology}

All colonic lesions were classified by the endoscopist according to the Paris classification [17]. Both polypoid and non-polypoid lesions, which were retrieved during colonoscopy, were processed and stained using standard methods and evaluated by an LHU-1 gastrointestinal pathologist (PI,CR). The pathologists were blinded to the allocation (SC or EAC group) of the patients. The lesions were evaluated according to the Vienna classification [18]. All lesions were classified as hyperplastic polyp, sessile serrated adenoma/polyp, serrated adenoma, tubular, tubulo-villous or villous adenoma or carcinoma. Dysplasia was defined as low- or high-grade. An advanced adenoma was defined as an adenoma $\geq 10 \mathrm{~mm}$ or an adenoma with $\geq 25 \%$ villous component or with high grade dysplasia.

\section{Study design, outcome measures and statistical analysis}

From 1 January to 31 December 2015 we performed consecutively EAC in all the subjects enrolled by our Department of Health responsible for the CRC screening program. Performance of EAC was compared with SC executed in the subjects enrolled by the same system from 1 January to 31 December 2014. The primary outcomes were: (a) adenoma detection rate 
(ADR) defined as the proportion of screening colonoscopies with at least one histological confirmed adenoma; (b) advanced adenoma detection rate (AADR) as the proportion of screening colonoscopies with at least one advanced adenoma; (c) mean number of adenomas per patient (MAP) defined as the total number of detected adenomas in each group divided by the total number of procedure in that group; (d) mean number of advanced adenomas per patient (MAAP); and (e) mean number of small $(<10 \mathrm{~mm})$ tubular adenomas per patient (MSTAP). Secondary outcomes were rates of cecal intubation and complications. Quantitative variables were expressed as mean, standard deviation and range. Categorical variables were presented as total numbers and percentages. A two-tailed chi-squared test for categorical variables and a Mann Whitney $U$ test for quantitative variables were used. A $P$ value $<0.05$ was considered statistically significant.

\section{Sample size}

The principal analysis of the study was to compare ADR between the EAC and SC groups. In our cohort, there were mainly two groups of patients: FIT-positives and those on surveillance for adenomas in a proportion of 80:20, respectively. Considering that the proportion of patients with at least one adenoma (ADR) would be $44 \%$ in FIT-positives [19] and $35 \%$ in patients on surveillance [20], an ADR of $42.2 \%$ for SC and an increase of $10 \%$ for EAC were assumed [12]. A minimum of 404 patients per group were required to achieve at least $80 \%$ power. Our screening colonoscopies/year are around 450 to 500 exams. To avoid selection bias, we decided arbitrarily to exceed the minimum required number of patients, offering EACs to all patients in 2015. Performance of EAC in 2015 was compared with that for SC in 2014. A type I error rate of $5 \%$, using two-sided tests was used. Statistical power analysis was performed using $G^{*}$ Power 3.17 [21].

\section{Results}

\section{Patient characteristics}

SC and EAC were performed in 579 and 605 CRC screening patients, respectively, consecutively enrolled into the study. To evaluate only the first colonoscopy, 33 and 86 patients who had repeated SC or EAC, respectively, for different reasons (e. g. to remove large polyps or to control the effectiveness of the endoscopic resection) were excluded. Therefore, 546 and 519 subjects that have undergone SC and EAC, respectively, were analyzed. They were comparable according to both age and gender. Indications for colonoscopy were similar between the two groups: $79.5 \%$ for FIT-positive, $18.1 \%$ for polyp surveillance and $2.4 \%$ to complete colonoscopy in the SC group; $81.1 \%$ for FIT-positive, $18.1 \%$ for polyp surveillance and $0.8 \%$ for completion in the EAC group $(P=0.1)$ ( $\triangleright$ Table 1$)$.

\section{Colonoscopy results}

Cecal intubation rate was $97.4 \%$ for SC and $97.1 \%$ forh EAC without significant differences. There was no difference in satisfactory bowel preparation (BBPS $\geq 6$ ) between SC and EAC. Mean withdrawal times were no different between the two groups nor were the percentages of patients for whom the procedure was performed under conscious or deep sedation or without sedation. Among the 59 patients (5.5\%) with an unsatisfactory BBPS (BBPS $<6)$, colonoscopy was completed in 26 patients $(76.5 \%)$ and in 17 patients $(68 \%)$ in the SC and EAC groups, respectively, with significant differences ( $\triangleright$ Table 2 ). Twenty-nine patients $(2.7 \%)$ had an incomplete colonoscopy because of several reasons ( $>$ Table 3 ).

\section{Complications}

In both groups, colonoscopies were performed without complications requiring early and late (within 1 month after the procedure) hospitalization.

\section{Colorectal lesion detection}

In the intention to-treat-analysis, ADR was $47.4 \%$ in SC and $52.4 \%$ in EAC with no statistically significant differences. AADR was $24.7 \%$ and $23.1 \%$ in SC and EAC respectively $(P=0.5)$. MAP significantly increased from $0.87(S D \pm 1.24$, range: $0-7)$ in $S C$ to 1.11 (SD \pm 1.55 , range: $0-13)$ in $\operatorname{EAC}(P=0.02)$. In the same way, MSTAP significantly increased from 0.57 (SD \pm 1.01 , range: $0-6)$ in SC to 0.81 (SD \pm 1.36 , range: $0-13)$ in $\operatorname{EAC}(P=0.01)$ but not in MAAP. Rates of CRC detection were $1.6 \%$ and $2.1 \%$ for SC and EAC, respectively $(P=0.6)(\triangleright$ Table 4$)$. Results were similar when the data were analyzed in per-protocol analysis (excluding both poor bowel preparation and uncompleted colonoscopy) ( $\triangleright$ Table 5).

\section{Discussion}

This study, performed in a large cohort of patients who underwent colonoscopy in an organized CRC screening regional program, showed that the Endocuff did not increase ADR and AADR. However, the device seemed to increase the number of adenomas - mostly for lesions $<10 \mathrm{~mm}$ - per patient, albeit the clinical relevance of this increase remains unclear. Furthermore, it was safe and it did not impair cecal intubation or withdrawal times.

Biecker et al., in the first multicenter randomized trial conducted in a mixed population, reported a significant increase in ADR (from $28 \%$ using SC to $36 \%$ with EAC) [11]. GonzálezFernández et al, in a recent randomized trial, confirmed a higher ADR in the Endocuff group (22\%) than in the SC one (13.5\%) [15]. Furthermore, a multicenter randomized tandem study showed a significantly lower miss rate of adenoma when Endocuff was used [16]. Conversely, Van Doorn et al., in a multicenter randomized trial performed in a large cohort of both asymptomatic and symptomatic subjects, did not find a difference in terms of ADR between SC and EAC. In particular, they observed a very high ADR in both groups (52\% in SC and EAC) [13]. ACcording to this result, a recent trial based on a fecal blood test CRC screening program found very high ADRs for both EAC and SC without achieving statistical difference (60 and $63 \%$ respectively) [14].

In our study, although a $5 \%$ increase in ADR in the EAC group ( $52 \%$ vs. $47 \%$ in SC) was obtained and it could be clinically significant, there was no statistically significant difference be- 
- Table 1 Patient characteristics and colonoscopy indication.

\begin{tabular}{|l|c|c|}
\hline & Standard colonoscopy $\mathbf{2 0 1 4}$ & Endocuff-assisted colonoscopy 2015 \\
\hline Number of colonoscopies & 546 & 519 \\
\hline Mean \pm SD (range) & $60 \pm 5.9(49-70)$ & $60 \pm 5.9(49-70)$ \\
\hline Gender M/F & $284 / 262(\mathrm{M} 51.4 \%)$ & $293 / 226(\mathrm{M} 56.5 \%)$ \\
\hline Colonoscopy indication & & \\
\hline - FIT-positive & $434(79.5 \%)$ & $421(81.1 \%)$ \\
\hline - Polyp surveillance & $99(18.1 \%)$ & $94(18.1 \%)$ \\
\hline - To complete colonoscopy ${ }^{1}$ & $13(2.4 \%)$ & $4(0.8 \%)$ \\
\hline $\begin{array}{l}\text { FIT, fecal immunochemical test. } \\
\text { 1 Due to poor bowel preparation or for reaching the cecum. }\end{array}$ & \\
\hline
\end{tabular}

- Table 2 Colonoscopy results.

\begin{tabular}{|l|c|c|c|}
\hline & Standard colonoscopy $\mathbf{2 0 1 4}$ & Endocuff-assisted colonoscopy 2015 & P value \\
\hline Number of colonoscopy & 546 & 519 & $05.36 \pm 2.07$ \\
\hline Withdrawal time (mean min \pm SD) & $05.57 \pm 2.21$ & $6 / 420 / 93$ & 0.5 \\
\hline Deep/conscious/without sedation & $5 / 456 / 85$ & $504(97.1 \%)$ & 0.7 \\
\hline Cecal intubation & $532(97.4 \%)$ & $15(2.9 \%)$ & 0.2 \\
\hline Incomplete colonoscopy & $14(2.6 \%)$ & $494(95.2 \%)$ & 0.3 \\
\hline BBPS $\geq 6$ & $512(93.8 \%)$ & $487 / 494(98.6 \%)$ \\
\hline Adjusted caecal intubation ${ }^{2}$ & $506 / 512(98.8 \%)$ & $17 / 25(68.0 \%)$ \\
\hline Cecal intubation in BBPS<6 & $26 / 34(76.5 \%)$ & 0.7 \\
\hline $\begin{array}{l}\text { BBPS, Boston Preparation Bowel Scale. } \\
\text { Adjusted cecal intubation for bowel preparation (patients with a poor preparation were excluded). }\end{array}$ \\
\hline
\end{tabular}

- Table 3 Reasons for incomplete colonoscopy.

\begin{tabular}{|l|l|l|l|}
\hline & Standard colonoscopy 2014 & Endocuff-assisted colonoscopy 2015 \\
\hline Incomplete colonoscopy & $14(2.4 \%)$ & $15(2.8 \%)$ & P value \\
\hline Poor bowel preparation & 8 & 8 \\
\hline Good bowel preparation & 6 & 7 \\
\hline - Benign strictures & 0 & 1 \\
\hline - Tortuous colon & 6 & 4 \\
\hline - Malignant stenosis & 0 & 2 \\
\hline
\end{tabular}

tween the two groups. This may be related to the high baseline ADR.

Furthermore, in our study, EAC detected significantly more small adenomas per patient. This is in line with Biecker et al. who detected more small adenomas per exam with Endocuff [12]. Van Doorn et al. found more diminutive $(<5 \mathrm{~mm})$ and flat adenomas with EAC as well [13]. Adenomas per colonoscopies have been proposed as another parameter of quality in addition to ADR because it may better discriminate between high and low adenoma detection than ADR $[22,23]$. Discovering more adenomas per exam may modify the classification of a patient's risk of metachronous lesions. According to the European Union CRC screening guidelines, in fact, if more than two small adenomas are both detected and removed, a patient shifts from low to intermediate class of risk, resulting in a reduced time to the next colonoscopy [24].

Another measure of quality of colonoscopy may be detection of serrated lesions. Detection of these lesions, which have different tumorigenesis, may not be proportional to ADR [25]. 
- Table 4 Polyp detection with standard colonoscopy and Endocuff-assisted colonoscopy. ${ }^{1}$

\begin{tabular}{|c|c|c|c|}
\hline & Standard colonoscopy 2014 & Endocuff-assisted colonoscopy 2015 & $P$ value \\
\hline Number of colonoscopies & 546 & 519 & \\
\hline Patients $\geq 1$ adenoma (ADR) & $259(47.4 \%)$ & $272(52.4 \%)$ & 0.1 \\
\hline Patients $\geq 1$ advanced adenoma (AADR) & $135(24.7 \%)$ & $120(23.1 \%)$ & 0.5 \\
\hline Patients $\geq 1$ serrated lesions (SDR) & $26(4.8 \%)$ & $35(6.7 \%)$ & 0.2 \\
\hline $\mathrm{MAP}($ mean $\pm S D$, range $)$ & $0.87 \pm 1.24(0-7)$ & $1.11 \pm 1.55(0-13)$ & 0.02 \\
\hline MAAP (mean $\pm S D$, range) & $0.30 \pm 0.58(0-3)$ & $0.29 \pm 0.59(0-3)$ & 0.6 \\
\hline MSTAP & $0.57 \pm 1.01(0-6)$ & $0.81 \pm 1.36(0-13)$ & 0.01 \\
\hline MSP (mean $\pm S D$, range) & $0.05 \pm 0.24(0-2)$ & $0.08 \pm 0.31(0-2)$ & 0.2 \\
\hline Colorectal cancer $n(\%)$ & $9(1.6 \%)$ & $11(2.1 \%)$ & 0.6 \\
\hline \multicolumn{4}{|c|}{$\begin{array}{l}\text { ADR, adenoma detection rate (percentage of patients with at least } 1 \text { adenoma); AADR, advanced adenoma detection rate (percentage of patient with at least } 1 \\
\text { advanced adenoma); SDR, serrated lesions detection rate (percentage of patients with at least } 1 \text { serrated lesion; MAP, mean number of adenomas per patient; MAAP, } \\
\text { mean number of advanced adenomas per patient; MSP, mean number of serrated lesions per patient; MSTAP, mean small (<10 mm) tubular adenomas per patient. } \\
{ }^{1} \text { All analyses are based on the intention-to-treat analysis. }\end{array}$} \\
\hline
\end{tabular}

- Table 5 Polyp detection with standard colonoscopy and Endocuff-assisted colonoscopy. ${ }^{1}$

\begin{tabular}{|c|c|c|c|}
\hline & Standard colonoscopy 2014 & Endocuff-assisted colonoscopy 2015 & $P$ value \\
\hline Number of colonoscopy & 506 & 487 & \\
\hline Patients $\geq 1$ adenoma (ADR) & $248(49.0 \%)$ & $265(54.4 \%)$ & 0.1 \\
\hline Patients $\geq 1$ advanced adenoma (AADR) & $130(25.7 \%)$ & $117(24.0 \%)$ & 0.5 \\
\hline Patients $\geq 1$ serrated lesions (SDR) & $25(4.9 \%)$ & $35(7.2 \%)$ & 0.1 \\
\hline MAP mean \pm SD (range) & $0.91 \pm 1.26(0-7)$ & $1.16 \pm 1.57(0-13)$ & 0.02 \\
\hline MAAP mean \pm SD (range) & $0.32 \pm 0.60(0-3)$ & $0.31 \pm 0.61(0-3)$ & 0.6 \\
\hline MSTAP mean \pm SD (range) & $0.60 \pm 1.04(0-6)$ & $0.86 \pm 1.39(0-13)$ & 0.001 \\
\hline MSP mean \pm SD (range) & $0.05 \pm 0.24(0-2)$ & $0.08 \pm 0.32(0-2)$ & 0.1 \\
\hline Colorectal cancer $n(\%)$ & $9(1.77 \%)$ & $9(1.84 \%)$ & 0.9 \\
\hline \multicolumn{4}{|c|}{$\begin{array}{l}\text { ADR, adenoma detection rate (percentage of patient with at least } 1 \text { adenoma); AADR, advanced adenoma detection rate (percentage of patient with at least } 1 \text { ad- } \\
\text { vanced adenoma); SDR, serrated lesions detection rate (percentage of patients with at least } 1 \text { serrated lesion); MAP, mean number of adenomas per patient; MAAP, } \\
\text { mean number of advanced adenoma per patient; MSTAP, mean small }(<10 \mathrm{~mm} \text { ) tubular adenomas per patient; MSP, mean number of serrated lesions per patient. } \\
{ }^{1} \text { All analyses are based on per-protocol analysis: poor bowel preparation }(\text { BBPS }<6) \text { and unreached cecum colonoscopies excluded. }\end{array}$} \\
\hline
\end{tabular}

In our study, Endocuff did not allow observation of more serrated lesions given their low occurrence.

We knowledge that the endoscopists were not blinded as in other studies [11-13]. Blinding was difficult for technical reasons, such as the visibility of the arms of the Endocuff and the small, but detectable, resistance during endoscopy. Thus, investigator-related bias cannot be ruled out as having contributed to results in favor of EAC.

However, the main limitation of our study is the lack of randomization. This is likely to be marginal. Irrespective of the design - i.e. randomized or not - the operator is not blinded to the intervention. Thus, randomization does not represent a means of preventing operator-related bias. The main aim of randomization is to ensure a balanced distribution of patients in the two arms, to prevent selection bias, i.e. patients at higher risk more prevalent in one of the two arms. However, such bias does not apply to an organized screening program for the following reasons. First, invitations are standardized per sex and age, ensuring homogeneous distribution across the years, as shown by the similar distribution between the two groups in the study. Second, FIT positivity represents by itself the highest risk factor for advanced neoplasia across colonoscopy indications, ensuring homogeneous enrichment of the population, as shown by the similar rate of advanced neoplasia between the two arms of the study. Marginalization of the role of randomization in ensuring a balance distribution of patients is indirectly confirmed by the lack of difference in main detection rates (ADR, AADR, SSP) between the two arms. 


\section{Conclusion}

In conclusion, although a $5 \%$ increase in EAC was seen in comparison to SC, our study did not achieve a statistically significant difference between the two groups. That result may be due to the high baseline ADR, which is related to the characteristics of the enrolled cohorts. Moreover, EAC detected significantly more small adenomas per patient.

Competing interests

None

\section{References}

[1] Ferlay J, Autier P, Boniol M et al. Estimates of the cancer incidence and mortality in Europe in 2006. Ann Oncol 2007; 18: 581 - 592

[2] Mandel JS, Bond TH, Church TR et al. Reducing mortality from colorectal cancer by screening for faecal occult blood. N Engl J Med 1993; 328: $1365-1371$

[3] Hardcasle JD, Chamberlain LO, Robinson MHE et al. Randomised controlled trial of faecal occult blood screening for colorectal cancer. Lancet 1996; 348: $1472-1477$

[4] Zorzi M, Fedeli U, Schievano E et al. Impact on colorectal cancer mortality of screening programmes based on the faecal immunochemical test. Gut 2015; 64: 784-790

[5] Zorzi M, Fedato C, Del Sole A et al. I programmi di screening oncologici del Veneto. Rapporto 2013-2014. 1st ed. Padova: CLEUP; 2015

[6] Hassan C, Repici A, Rex DK. FITting ADR to colonoscopy indication. United European Gastroenterol J 2017; 5: 149-152

[7] Rex DK, Schoenfeld PS, Cohen J et al. Quality indicators for colonoscopy. Gastrointest Endosc 2015; 81: 31-53

[8] Corley DA, Jensen CD, Marks AR et al. Adenoma detection rate and risk of interval cancer. N Engl J Med 2014; 370: 1298-1306

[9] Konda V, Chauhan SS, Abu Dayyeh Barham K et al. Endoscopes and devices to improve colon polyp detection. Gastrointest Endosc 2015; 81: $1122-1129$

[10] Lenze F, Beyna T, Lenz P et al. Endocuff-assisted colonoscopy: a new accessory to improve adenoma detection rate? Technical aspects and first clinical experience Endoscopy 2014; 46: 610-614

[11] Biecker E, Floer M, Heinecke A et al. Novel Endocuff-assisted colonoscopy significantly increases the polyp detection rate: a randomized controlled trial. J Clin Gastroenterol 2015; 49: 413-418
[12] Floer M, Biecker E, Fitzlaff $R$ et al. Higher adenoma detection rates with Endocuff-assisted colonoscopy - a randomized controlled multicenter trial. PLoS One 2014; 9: e114267

[13] Van Doorn SC, van der Vlugt M, Depla A et al. Adenoma detection with Endocuff colonoscopy versus conventional colonoscopy: a multicentre randomized controlled trial. Gut 2017; 66: 438 - 445

[14] Bhattacharyya R, Chedgy F, Kandiah K et al. Endocuff-assisted vs. standard colonoscopy in the fecal occult blood test-based UK Bowel Cancer Screening Programme (E-cap study): a randomized trial. Endoscopy 2017; 49: $1043-1050$

[15] González-Fernández C, García-Rangel D, Aguilar-Olivos NE et al. Higher adenoma detection rate with the endocuff: a randomized trial. Endoscopy 2017; 49: 1061 - 1068

[16] Triantafyllou K, Polymeros D, Apostolopoulos P et al. Endocuff-assisted colonoscopy is associated with a lower adenoma miss rate: a multicenter randomized tandem study. Endoscopy 2017; 49: 1051 1060

[17] The Paris endoscopic classification of superficial neoplastic lesions: esophagus, stomach, and colon. November 30 to December 1, 2002. Gastrointest Endosc 2003; 58: S3 - S43

[18] Schlemper R], Riddell RH, Kato Y et al. The Vienna classification of gastrointestinal epithelial neoplasia. Gut 2000; 47: 251-255

[19] Zorzi M, Senore C, Da Re F et al. Quality of colonoscopy in an organised colorectal cancer screening programme with immunochemical faecal occult blood test: the EQuIPE study (Evaluating Quality Indicators of the Performance of Endoscopy). Gut 2015; 64: 1389-1396

[20] Kahi C], Vemulapalli KC, Johnson CS. Improving measurement of the adenoma detection rate and adenoma per colonoscopy quality metric: the Indiana University experience. Gastrointest Endosc 2014; 79: $448-454$

[21] Faul F, Erdfelder E, Lang AG et al. G* Power 3: a flexible statistical power analysis program for the social, behavioral, and biomedical sciences. Behav Res Methods 2007; 39: 175-191

[22] Wang HS, Pisegna J, Modi R et al. Adenoma detection rate is necessary but insufficient for distinguishing high versus low endoscopist performance. Gastrointest Endosc 2013; 77: 71 - 78

[23] Denis B, Sauleau EA, Gendre I. Measurement of adenoma detection and discrimination during colonoscopy in routine practice: an exploratory study. Gastrointest Endosc 2011; 74: 1325-1336

[24] Atkin WS, Valori R, Kuipers EJ et al. European guidelines for quality assurance in colorectal cancer screening and diagnosis. First Edition Colonoscopic surveillance following adenoma removal. Endoscopy 2012; 44: $151-163$

[25] Kahi C], Hewett DG, Norton DL et al. Prevalence and variable detection of proximal colon serrated polyps during screening colonoscopy. Clin Gastroenterol Hepatol 2011; 9: 42-46 